\title{
ÍNDICE DE INCERTIDUMBRE DE POLIITICA ECONÓMICA PARA ECUADOR: DISCUSIONES Y UNA PROPUESTA DE CUANTIFICACIÓN
}

\section{UNCERTAINTY INDEX OF ECONOMIC POLICY OF ECUADOR: DISCUSSIONS AND A PROPOSAL FOR QUANTIFICATION}

SEbastián PADilla ${ }^{1}$

Recibido: 12 de abril

Aceptado: 1 de mayo 



\title{
ÍNDICE DE INCERTIDUMBRE DE POLIITICA ECONÓMICA PARA ECUADOR: DISCUSIONES Y UNA PROPUESTA DE CUANTIFICACIÓN
}

\section{UNCERTAINTY INDEX OF ECONOMIC POLICY OF ECUADOR: DISCUSSIONS AND A PROPOSAL FOR QUANTIFICATION}

\begin{abstract}
Sebastián Padilla
Palabras clave: Incertidumbre, Política Económica, Noticias, Ecuador

Keywords: Uncertainty, Economic Policy, News, Ecuador

\section{RESUMEN}

En este trabajo se construye el primer índice de incertidumbre de política económica para Ecuador. Como parte de una reflexión previa se presentan discusiones teóricas acerca del rol de la incertidumbre en el proceso de toma de decisiones y la validez de asignar probabilidades para estimar el futuro. El resultado es una variable proxy de frecuencia mensual desde el año 1999 al 2017 con información del periódico "El Comercio". La metodología sigue la propuesta de Baker, et al. (2016) en base al conteo de noticias con palabras clave. El índice parece reflejar momentos en la historia reciente con altos niveles de incertidumbre reconocidos a discreción. En una comparación con otras medidas de incertidumbre y posibles indicadores anticipados se encontraron correlaciones significativas. 


\begin{abstract}
This paper presents the first economic policy uncertainty index for Ecuador. As part of a previous reflection, theoretical discussions on the role of uncertainty on the decision-making process and the validity of allocating probabilities to estimate the future are presented. The resulting index is a continuous proxy variable of monthly frequency comprising the periods between 1999

Comercio" newspaper. The methodology follows the proposal of Baker, et al. (2016) based on counting news articles containing specific keywords. The index seems to reflect moments in recent history with high levels of uncertainty recognized at discretion. In a comparison with other measures of uncertainty and possible anticipated indicators, significant correlations were found.
\end{abstract} and 2017 with information from the "El

\title{
JEL No: C43, D81, E20
}

\section{INTRODUCCIÓN}

En este trabajo se construye el primer índice de incertidumbre de política económica para Ecuador. El concepto al que se hace referencia como incertidumbre fue propuesto por Knight (1921) y discutido también por Keynes $(1936,1937)$. Esta incertidumbre genuina o knightiana no se puede computar debido a la naturaleza impredecible de los sistemas económicos.

La incertidumbre afecta a las decisiones de inversión, preferencia por liquidez (Keynes, 1936) e indirectamente al crecimiento y el nivel de empleo (Bloom, 2009). La falta de una medida o aproximación de la incertidumbre no permite que se pueda estudiar su trayectoria, así como tampoco estimar su impacto en el desempeño de una economía.

Con el fin de comprender de mejor manera la incertidumbre, se presenta un debate entre diversas teorías económicas del siglo XX acerca del papel de la incertidumbre en la formación de expectativas, el proceso de toma de decisiones y específicamente la validez de asignar probabilidades para estimar el futuro.

Para tal efecto se enfrentan los argumentos de la hipótesis de expectativas racionales, la teoría de la utilidad esperada y la teoría post keynesiana desde los trabajos originales y obviando recurrir 
a trabajos de recopilación para evitar sesgos en la presentación de la información.

Para la construcción del índice, la metodología precursora de este tipo de trabajos utiliza la frecuencia de artículos que contienen palabras clave en 10 periódicos de diversas líneas editoriales en Estados Unidos. En Latinoamérica, Cerda, et al. (2018) presentan el índice para Chile con noticias del diario El Mercurio desde el año 1993 y Perico (2018) realiza lo mismo para Colombia con noticias del periódico El Tiempo desde el año 1994.

El resultado de este trabajo es una variable proxy de frecuencia mensual desde el año 1999 al 2017 con información del periódico "El Comercio". Aunque la idea de generalizar una percepción resulta imposible, realizar una aproximación o propuesta es una tarea menos complicada. Tanto la metodología propuesta como la fuente de datos cumplen su función de captar, de alguna forma, los insumos de información que reciben los individuos.

El índice construido muestra valores elevados de incertidumbre, por sobre dos veces la media, en cuatro momentos desde al año 1999. El primero de ellos es el feriado bancario y la consecuente dolarización del sistema monetario, eventos que se anunciaron en marzo del 1999 y enero del 2000 respectivamente; un segundo momento a inicios del 2003 con varios eventos como posibles precursores de incertidumbre como la oposición a la construcción del OCP y el fracaso del CONAM; un tercer evento en 2008 cuando tuvo lugar la crisis financiera que golpearía a las economías latinoamericanas en 2009; el cuarto evento reflejado en el índice es la caída del precio de petróleo que inició en 2015 y que entre varias consecuencias en la economía ecuatoriana llevó a una recesión en el año 2016.

Finalmente, en una comparación con otras medidas de incertidumbre se encontraron correlaciones positivas significativas que van desde 0,24 hasta 0,50, una correlación negativa significativa con un rezago del precio del petróleo $(-0,19)$ y la inexistencia de una correlación con la variación de depósitos a la vista que se esperaría fuera negativa siguiendo la teoría keynesiana de un aumento de la preferencia por la liquidez en momentos de incertidumbre.

\section{EFECTOS DE LA INCERTIDUMBRE EN LA ECONOMÍA}

Una de las primeras referencias a la incertidumbre fue propuesta por
Knight (1921) como parte de su trabajo sobre riesgo, incertidumbre y ganan- 
cia. En su trabajo, basado en decisiones empresariales, argumenta que la incertidumbre hace referencia a situaciones no probabilísticas en las que los individuos no pueden saber toda la información requerida del futuro y deciden en un contexto incierto.

Esta definición, se diferencia del riesgo que corresponde a situaciones probabilísticas y sobre las que se ha trabajado hasta la actualidad en modelos macroeconómicos, financieros y microeconómicos modernos como la nueva economía industrial.

Extrapolar la incertidumbre de la producción empresarial hacia un nivel agregado fue una de las ideas de Keynes (1936) quien sugirió que la inversión es el componente más volátil de la demanda agregada, precisamente porque se basa en gran medida en opiniones acerca de eventos futuros, los cuales son necesariamente inciertos.

Al respecto Keynes (1937) en un artículo que discutía las críticas a su trabajo de 1936 hace la siguiente definición de incertidumbre:

Por conocimiento "incierto", permítanme explicar, no me refiero simplemente a distinguir lo que se conoce con certeza de lo que es solo probable. ... El sentido en el que estoy usando el término es aquel en el que la perspectiva de una guerra europea es incierta, o el precio del cobre y la tasa de interés dentro de veinte años... Sobre estos asuntos no existe una base científica sobre la cual se pueda calcular ninguna probabilidad calculable. Simplemente no sabemos' (Keynes, 1937)

Para Keynes (1936) la inversión está determinada por la tasa de interés esperada y por la eficiencia marginal del capital, esta última se ve afectada en mayor medida por la incertidumbre. Esta incertidumbre afecta la función de la inversión en el núcleo de su motivación y está sujeta a una estimación subjetiva que oscila entre el optimismo y el pesimismo, afectando en último término, según Keynes, a la demanda de inversión.

Más adelante, Bernanke (1983) argumentará que la incertidumbre retarda la tasa de inversión porque incrementa el valor de esperar por nueva información que permita continuar o concluir la inversión. Bernanke es el primer autor en realizar un modelo que relacione la incertidumbre y la inversión; en el mismo supone que la inversión puede ser diferida y dependerá del esclarecimiento de la información y del estado de la inversión. En este sentido, según Bernanke

${ }^{1}$ Traducción propia. 
(1983), la incertidumbre que motiva a dilatar las decisiones de inversión da como resultado una postergación de los incrementos en la producción y las decisiones de contratación, afectando así al crecimiento económico y al empleo.

Bloom (2009) sigue el argumento de Bernanke y concluye que, bajo la circunstancia de un shock de incertidumbre con importantes costos irreversibles, conocido como real options effect, la mejor opción de los inversionistas es esperar y ver (wait-and-see option). Cuando el shock de incertidumbre se disipa y las condiciones del negocio vuelven a ser óptimas, las firmas se encuentran con un nivel de capital y trabajo lejos del óptimo.

A diferencia de los hallazgos en países desarrollados que siguen la metodología de Bloom (2009), Carriére-Swallow \& Céspedes (2013) realizaron un estudio diferenciado entre países en vías de desarrollo y desarrollados; hallaron que en promedio los primeros exhiben mayores caídas de inversión, mayor tiempo de recuperación y una caída más fuerte en consumo privado frente a un shock de incertidumbre.

Otro estudio en el que la incertidumbre se ve relacionada con variables reales es el llevado a cabo por Arellano, et. al (2016), en el cual la incertidumbre contribuye al llamado risk premium effect. Este efecto hace referencia a que la incertidumbre eleva el riesgo tanto de los créditos privados como el de los públicos - la contratación de deuda pública-. En escenarios de alta incertidumbre los bancos contraen el crédito para proteger su patrimonio y si no lo hacen aumentan la tasa de interés; en estas circunstancias se vuelve más difícil iniciar un proyecto.

\section{DISCUSIONES TEÓRICAS SOBRE INCERTIDUMBRE}

Quizás la arista más importante al tratar la incertidumbre tiene que ver con las decisiones cuando esta situación se percibe, cómo los individuos toman esas decisiones y específicamente la validez de asignar probabilidades para estimar el futuro. En ese sentido, el punto de partida más importante es el debate propuesto por Davidson (1991) en refe- rencia al trabajo de Machina (1987), el cual es un gran insumo de información sobre el tema a tratar para luego ahondar en trabajos similares y enfrentar las diferentes concepciones teóricas.

Davidson (1991), presenta una visión post keynesiana de la incertidumbre iniciando con una crítica a la teoría dominante: 
"Las perspectivas mainstream relacionadas a la incertidumbre suponen que las expectativas están basadas ya sea en un análisis estadístico de datos pasados con señales de mercado proveyendo información acerca de probabilidades objetivas o en percepciones subjetivas de estas probabilidades fundadas en los axiomas de la teoría de la utilidad esperada" (Davidson, 1991)².

Finalizando su trabajo, da luces sobre el tema a discutir al referir que las perspectivas del mainstream ilustrado por Machina (1987) no pueden lidiar con casos en los que existe incertidumbre real. La teoría ortodoxa o mainstream con relación a la incertidumbre, sigue en unos casos los preceptos de la hipótesis de expectativas racionales y en otros la teoría de la utilidad esperada.

Por otro lado, la teoría post keynesiana en palabras de Davidson (1991) ha desarrollado una perspectiva diferente, en la que las distribuciones de probabilidad que fundan las dos teorías anteriores no son la base para comprender el comportamiento del mundo real bajo incertidumbre.

En su trabajo, Davidson (1991) presenta una clasificación de lo que él llama ambientes de toma de decisiones mutuamente excluyentes: ambiente de probabilidad objetiva, ambiente de probabilidad subjetiva y ambiente de incertidumbre real. Cada uno de ellos hace referencia a la formación de distribuciones de probabilidad bajo la óptica de la hipótesis de expectativas racionales, teoría de la utilidad esperada y la perspectiva post keynesiana respectivamente.

\section{Probabilidad objetiva: Hipótesis de expectativas racionales}

Muchas de las ideas, modelos y aportes teóricos que utilizan la hipótesis de expectativas racionales se encuentran compilados en el libro "Rational Expectations and Economic Practice" editado por Lucas y Sargent en 1981. Entre ellos, destacan los aportes de John Muth quien fue el primero en proponer la hipótesis de expectativas racionales en oposición a la teoría de expectativas adaptativas.

Para Muth (1961), las expectativas —debido a que son predicciones informadas sobre futuros eventos - son esencialmente lo mismo que las predicciones de la teoría económica relevante.

"Las expectativas de las firmas (o de manera general, la distribución de probabilidad subjetiva de los resultados) tiende a estar distribuida, para el mismo conjunto de información, al-

\footnotetext{
${ }^{2}$ La gran mayoría de las citas e ideas de los autores mencionadas en este trabajo son traducciones propias.
} 
rededor de la predicción de la teoría (o la distribución de probabilidad objetiva de los resultados)" (Muth, 1961)

La hipótesis afirma tres cosas: 1) La información es escaza y el sistema económico generalmente no la desperdicia. 2) La manera en que las expectativas están formadas dependen específicamente de la estructura del sistema relevante que describe la economía. 3) Una predicción pública no tendrá efecto sustancial en la operación del sistema económico. Además, menciona que las expectativas de una sola firma pueden estar erradas de la teoría, "...la hipótesis no afirma que el cálculo de los empresarios se acerque al resultado del sistema de ecuaciones en ningún sentido o que las predicciones de empresarios son perfectas" (Muth, 1961).

Esta hipótesis se volvió esencial en los modelos macroeconómicos durante gran parte de los años 70 y 80 . Sargent \& Wallace (1976) dan luces sobre las razones para utilizar la hipótesis en los modelos:

“... está de acuerdo con la práctica usual de los economistas de asumir que las personas se comportan buscando sus mejores intereses. No se intenta negar que algunas personas son irracionales y neuróticas, pero no tenemos razones para creer que esas irracionalidades causen desviaciones sistemáticas y predecibles del comportamiento racional, el cual puede ser modelado por un macroeconomista..." (Sargent \& Wallace, 1976)

La hipótesis de expectativas racionales no requiere que las expectativas de la gente sean iguales a expectativas matemáticas condicionales, tan solo que se igualen a las expectativas matemáticas condicionales más un gran término aleatorio (aleatorio con respecto a la información condicionante) de la forma:

$$
E_{t-1} p^{*}{ }_{t}=E_{t-1} p_{t}+\varnothing_{t}
$$

En donde, $E_{t-1} \varnothing_{t}=0$ y $\varnothing_{t}$ es un término aleatorio que permite desviaciones aleatorias grandes respecto a la racionalidad (Sargent \& Wallace, 1976).

Para entender de mejor manera la hipótesis, en un trabajo previo, los mismos autores otorgan luces sobre la distribución de probabilidades que se imponen a los modelos para que cumplan con la hipótesis de expectativas racionales. El requisito para que las expectativas del público sean racionales es que:

$$
E_{t+1} p^{*}{ }_{t-j}=E_{t-j} p_{t+i}
$$

En donde $E_{t-j} p_{t+i}$ es la esperanza matemática de $p_{t+i}$ calculada usando el modelo (probabilidad de distribución de 
$\left.p_{t+i}\right)$ y toda la información que se asume disponible al final del período $t-j$ La información disponible se asume que es consistente con los datos en el presente y los valores pasados de todas las variables endógenas y exógenas observadas al final del período $t$-j, esto es $\varnothing^{*}{ }_{t-j}$. (Sargent \& Wallace, 1975).

Blanchard et al. (2012) afirman que las expectativas racionales a diferencia de las adaptativas, no son el resultado de sencillas reglas basadas en el pasado. Las expectativas racionales suponen que la gente observa el futuro, en el sentido de que utiliza la información que tiene de la mejor manera y hacen todo lo posible por predecirlo.

Es interesante replicar que para Blanchard et al. (2012), "Existen momentos en que la gente, las empresas o los mercados financieros pierden de vista la realidad y se muestran excesivamente optimistas o pesimistas, pero estos casos son más la excepción que la regla y no está claro que los economistas puedan decir mucho sobre estos momentos" (Blanchard, et al., 2012).

Davidson (1991), sin embargo dirá que las expectativas racionales se cumplen solo cuando existen en un mundo ergódico racional, es decir en el que el pasado se comporte como el futuro. Existe evidencia empírica (Danziger, et al., 1982) —a ser desarrollada más adelante- de que el comportamiento aun- que parezca ser en gran parte irrracional al verlo desde el supuesto de la existencia de un mundo ergódico racional, es en realidad completamente coherente con el mundo real.

Blanchard et al. (2012) al resumir la hipótesis de las expectativas racionales, a diferencia de Davidson (1991), la trata como normativa más que descriptiva. Blanchard defiende la idea de que el supuesto se utiliza como hipótesis de trabajo mas no porque los economistas crean que la gente siempre tiene expectativas racionales.

\section{Probabilidad subjetiva: Teoría de la utilidad esperada y Teoría prospectiva}

Una importante fuente de información sobre teorías de decisión, entre ellas la teoría de la utilidad esperada, se encuentra disponible en la "Enciclopedia de Filosofía de Stanford". Dicha fuente de información al referirse a la teoría de la utilidad esperada especifica que, aunque no modela de manera certera los procesos psicológicos de toma de decisiones, pretende predecir las elecciones de la gente.

La utilidad esperada de un acto, en palabras de Briggs (2017), es un promedio ponderado de las utilidades de cada uno de los posibles resultados en el que la utilidad de un resultado mide la magnitud en el que ese resultado es 
preferido por sobre las demás alternativas. Esa utilidad, es medida de acuerdo a la probabilidad de que el acto conlleve al resultado.

La teoría y tantos trabajos posteriores sobre la misma, así como las críticas, hacen uso de términos específicos que se pudieran traducir como actos, estados y resultados ${ }^{3}$. Los actos son objetos de las preferencias instrumentales bajo el mando de quien toma la decisión (llevar o no paraguas por si llueve). Los estados son cosas fuera del control de quien toma la decisión que pueden influir en el resultado (llueve o no llueve). Los resultados son objetos de preferencias no instrumentales (terminar seco, seco con el paraguas en mano o mojado) (Briggs, 2017).

Con estas consideraciones, la utilidad esperada del acto $A$ de manera formal se expresa como:

$$
E U(A)=\sum_{0 \in O} P_{A}(O) U(0)
$$

En donde $O$ es el conjunto de resultados, $P_{A}(0)$ es la probabilidad del resultado $O$ condicional al acto $A$ y $U(0)$ es la utilidad de o (Briggs, 2017). Por lo tanto, en la teoría de la utilidad esperada, la utilidad esperada de un acto depende del valor de cada resultado (esto medido por la utilidad) y de la probabilidad de cada resultado condicional al acto, de todo el conjunto de resultados posibles.

La importancia para comprender de mejor manera las decisiones bajo incertidumbre recae sobre el tratamiento a las probabilidades. En este sentido, la propuesta de tratar a las probabilidades como grados de convicción o preferencias (Savage, 1954) parece ser la que mejor resume la idea de probabilidades subjetivas citada tanto por Machina (1987), Briggs (2017) y criticada por Davidson (1991, 2002).

Los actos son objetos de utilidad esperada pero no de preferencia intrínseca (se es indiferente entre llevar o no paraguas a menos que piense en salir); los estados son conjuntos de los estados anteriores en el sentido de que la probabilidad de cada estado es subjetiva al individuo (llueve, hace sol o llueve, nieva o llueve o hace sol, hace sol o nieva, nieva, etc.); los resultados son objetos de preferencia intrínseca y utilidad independientemente del estado (se prefiere estar seco así llueva, haga sol o nieve).

Para Savage (1954), ningún estado dirige un acto y son en conjunto, el acto más el estado los que determinan el resultado. Importante es también el supuesto de que, para cada resultado, hay un acto que conlleva a tal resultado independientemente del estado. "Al

\footnotetext{
${ }^{3}$ En el texto de Briggs (2017) se los llama acts, states y outcomes respectivamente.
} 
decidir sobre un acto, se debe tomar en cuenta todos los posibles estados del mundo, y las consecuencias implícitas de cada acto para cada posible estado del mundo" (Savage, 1954).

El autor postula una relación de preferencias sobre los actos y presenta axiomas sobre cómo se maneja esa relación, esto permite definir probabilidades subjetivas o grados de convicción con la relación "al menos tan probable como". Definir esa relación entre preferencias es la base del postulado de Savage. Tal relación subjetiva puede ser representada por una única función de probabilidad que engloba el proceso de toma de decisiones:

$$
P(E) \geq P(F)
$$

Se elige el acto que lleve al mejor resultado en un estado $E$ si y solo si el estado $E$ es al menos tan probable como el estado $F$.

Al finalizar su trabajo, Savage sin embargo aclara que "una persona puede no conocer todas las consecuencias de los actos en cada estado del mundo... la tarea no es ni remotamente posible para la capacidad humana... puede ser utilizado cuando se ataca a problemas de decisión relativamente simples" (Savage, 1954).

El orden de preferencias propuesto por Savage (1954) implica según Da- vidson $(1991,2002)$ que existe un conjunto finito de actos y resultados, y que cada agente puede realizar un ordenamiento de preferencias transitivo y completo de todas las posibles alternativas de elección lo cual está por fuera de la capacidad de cómputo humana.

\section{Incertidumbre real: Teoría post keynesiana}

Para Davidson (1994) existen al menos dos proposiciones desde el punto de vista metodológico que son relevantes para el desarrollo del análisis de la economía y de la política económica: 1) la distinción entre riesgo e incertidumbre y 2) el carácter no ergódico (no predecible) del entorno económico.

Con relación a la primera proposición, Keynes (1937) en un artículo que discutía las críticas a su "Teoría General del Empleo, el Interés y el Dinero" de 1936 hace la siguiente definición de incertidumbre:

Por conocimiento "incierto", permítanme explicar, no me refiero simplemente a distinguir lo que se conoce con certeza de lo que es solo probable. ... El sentido en el que estoy usando el término es aquel en el que la perspectiva de una guerra europea es incierta, o el precio del cobre y la tasa de interés dentro de veinte años... Sobre estos asuntos 
no existe una base científica sobre la cual se pueda calcular ninguna probabilidad. Simplemente no sabemos" (Keynes, 1937)

Según Davidson (2002), la razón por la que Knight (1921) y Keynes (1936) propusieron diferenciar entre riesgo e incertidumbre se debió a que la teoría neoclásica durante el siglo XIX e inicios del siglo XX al tratar sobre la incertidumbre había recuperado la noción ricardiana, conocida como presunción de conocimiento previo perfecto.

Esta se refería a la diferencia entre el valor estimado de un evento futuro y el valor de un evento perfectamente determinado que evoca el mismo comportamiento. El hecho de asignar una distribución de frecuencia dio paso a que tal probabilidad intente ser estimada.

Ya al hablar sobre la teoría macroeconómica keynesiana, para Ferrari-Filho \& Conceicao (2005), el legado principal de Keynes consiste en demostrar la lógica de una economía monetaria en la que las fluctuaciones en la demanda efectiva y el empleo ocurren porque, en un mundo con un futuro incierto y desconocido, los individuos prefieren retener dinero posponiendo las decisiones de consumo e inversión.

En ella, el dinero tiene tres propiedades: 1) la elasticidad de producción del dinero es cero; 2) la elasticidad de sustitución del dinero es cero; 3) el costo de transporte del dinero es cero. Estas propiedades dan paso a dos propósitos del dinero en una economía: 1) medio de circulación y 2) reserva de valor.

La función de reserva de valor provee seguridad ante la incertidumbre y une el pasado, el presente y el futuro. La no neutralidad del dinero hace que, en condiciones de incertidumbre, la demanda efectiva sea insuficiente porque los individuos prefieren mantener el dinero en lugar de comprar o invertir (preferencia por la liquidez). Ante la imposibilidad de modelar la incertidumbre de una manera determinística en una economía monetaria, las decisiones serán hechas en base a convenciones (Ferrari-Filho \& Conceicao, 2005).

Para Keynes (1936) y Davidson (1994), la existencia de incertidumbre explica la volatilidad de la inversión y a su vez la racionalidad de la preferencia por la liquidez, que causa fluctuaciones en la demanda efectiva y el empleo. Las decisiones de inversión envuelven convenciones e intuiciones, que desde el lado de los empresarios los obligan a apelar a sus instintos o en palabras de Keynes, animals spirits.

En referencia a la segunda proposición, el axioma ergódico supone que la única manera de que la gente observe el futuro utilizando la información que tiene de la mejor manera para predecirlo (hipó- 
tesis de expectativas racionales) es si los individuos creen que el pasado es estadísticamente confiable para que, a través de una muestra se convierta en una guía del futuro y así ayude a tomar decisiones en el presente (Davidson, 1991).

La teoría post keynesiana frente a la incertidumbre postula que tanto las decisiones en un ambiente objetivo en referencia a los modelos de hipótesis de expectativas racionales, como las decisiones en un ambiente subjetivo en referencia a los axiomas de la teoría de utilidad esperada, hacen eco de distribuciones de probabilidad que no son la base para comprender el comportamiento del mundo real bajo incertidumbre (Davidson, 1991).

En relación a la línea de la hipótesis de expectativas racionales, Davidson (2002) explica que la intención de medir la incertidumbre en términos de una distribución de probabilidad implica asumir que los datos existentes son parte de una serie de tiempo generada por un proceso estocástico ergódico, lo cual significa que "el proceso estocástico genera probabilidades objetivas inmutables que gobiernan los datos del pasado, el presente y el futuro" (Davidson, 2002).

Las expectativas para Davidson (1991, 2002) solo son racionales en un mundo ergódico hipotético que no distingue el paso del tiempo en el que el proceso económico es uniforme y consistente." Todos los modelos de expectativas racionales requieren el axioma ergódico como fundamento lógico de que todas las relaciones económicas son leyes naturales de movimiento que han sido preprogramadas en el sistema en el instante inicial de su creación" (Davidson, 2002).

Un ejemplo de hallazgos de que la formación de expectativas es completamente coherente con el mundo real, aunque parezca irrracional al verlo desde un mundo ergódico racional, es el estudio de Danziger, et al. (1982). En este estudio llevado a cabo en 9000 hogares, los investigadores encontraron que los ancianos gastan menos que los adultos con un mismo nivel de ingreso y que la persona más vieja del grupo de ancianos tenía la propensión a consumir más baja.

Si los individuos del estudio decidirían en un mundo ergódico según la hipótesis de las expectativas racionales, deberían haber mostrado un patrón de consumo según la teoría del ciclo de vida en el que las pesonas ahorran para sus años de jubilación y luego gastan su riqueza acumulada a medida que envejecen (Danziger, et al., 1982).

Los autores concluyen que los individuos del estudio enfrentan un complejo problema de incertidumbre sobre su salud, esperanza de vida, y capacidad para mantener hogares independientes 
y su respuesta es reducir su consumo incrementando su propensión a ahorrar a todos los niveles de ingreso durante su jubliación (Danziger, et al., 1982).

\section{MEDICIÓN DE LA INCERTIDUMBRE DE POLÍTICA ECONÓMICA}

El método propuesto por Baker, et al. (2016) de conteo de noticias con palabras relacionadas con incertidumbre ha generado un gran número de trabajos, alrededor de 20 índices de países diferentes. La literatura empírica se ha basado principalmente en variables proxy. Una variable proxy es una variable observada relacionada, pero no idéntica a una variable explicativa inobservable en el análisis de regresión múltiple (Wooldridge, 2012).

Dentro de los estudios con variables proxy se encuentran las que utilizan la volatilidad de los índices de retorno de mercado de valores (Bloom, 2009); la dispersión de datos de sección cruzada de ganancias de empresas, de retornos de acciones o de productividad (Bachmann, et al., 2013); la dispersión de datos de sección cruzada de pronósticos profesionales (D'Amico \& Orphanides, 2008) y finalmente los índices basados en conteo de noticias con palabras relacionadas con incertidumbre (Baker, et al., 2016). Para Jurado, et al. (2015) estas medidas basadas en variables proxy tienen la ventaja de ser directamente observables, pero pueden no estar correlacionadas con el proceso estocástico a ser analizado.

La construcción del índice de incertidumbre de política económica para el Ecuador se realizó con los datos obtenidos de manera directa y presencial en el archivo del periódico El Comercio, uno de los productos de Grupo El Comercio. Las noticias publicadas por el diario se encuentran digitalizadas en formato .pdf desde el año 1906 y en diversas plataformas digitales de gestión editorial y de archivo.

Desde el año 1995 al 2003 las noticias se almacenaban en un programa llamado Media Grid, en el año 2001 se adquirió el programa gn 3 Editorial, sistema editorial con diversos módulos entre los que se encuentra The Shell, programa en el que se encuentran todos los artículos desde el año 2001 al 2013. A este programa se realizó una transferencia de archivos desde Media Grid con los artículos del año 1999 y 2000; aunque tuvo éxito la copia realizada, se presentó un conflicto con las fechas, lo que retrasó un poco el conteo de noticias en estos dos años.

En el año 2014 se empezó a utilizar una tercera plataforma Ilamada Xalok 
la cual contiene los artículos publicados desde 2014 hasta la fecha. Debido a la periodicidad propuesta de la investigación se realizó la búsqueda y conteo de artículos en The Shell y Xalok, con la guía de dos personas encargadas del archivo.

Siguiendo la metodología propuesta se realizaron búsquedas de artículos que contenían palabras dentro de tres categorías: Economía, Política e Incertidumbre. Al índice con noticias dentro de estas tres categorías se lo llamó EPU por sus siglas en inglés. El índice es una serie mensual de 228 observaciones desde enero de 1999 a diciembre de 2017. Cada observación corresponde al conteo de artículos escritos durante un mes que contenían palabras claves dentro de cada una de las categorías $\mathbf{E}, \mathbf{P}, \mathbf{U}$.

En la siguiente tabla se indican las palabras clave utilizadas por categoría en la búsqueda:

\section{Tabla 1: Palabras clave por categoría}

\begin{tabular}{|ll|}
\hline Categoría & Palabras \\
\hline $\begin{array}{c}\mathbf{E}=\text { Economía } \\
+\end{array}$ & $\begin{array}{l}\text { Cualquier palabra que empiece con econ* (puede ser econo- } \\
\text { mista, economía, económica, etc.) }\end{array}$ \\
\hline $\begin{array}{c}\mathbf{P}=\text { Política Económica } \\
+\end{array}$ & $\begin{array}{c}\text { Al menos una palabra de las siguientes: política o impuesto o } \\
\text { congreso o asamblea o público o déficit o fiscal, o presupues- } \\
\text { to o banco central o finanzas o crisis o deuda }\end{array}$ \\
\hline $\mathbf{U}=$ Incertidumbre & Incertidumbre \\
\hline
\end{tabular}

Elaborado por: Sebastián Padilla

De esta manera, para que un artículo sea considerado parte de la frecuencia mensual debe contener al menos una de las palabras por categoría y tres en total por noticia escrita. En la construcción de la base de datos dentro de los programas mencionados, se siguió un orden en el que el primer criterio de búsqueda fue definir el año a buscar. En segundo lugar se colocaron las pala- bras "econ* AND incert*", el operador $\left(^{*}\right)$ permite al programa buscar cualquier

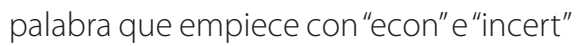
y así evitar tener que realizar combinaciones de búsqueda y disminuir el tiempo empleado en ello.

Aunque la palabra"incierto"en un inicio formaba parte de la categoría U, al combinarla con econ* la frecuencia resultaba muy pequeña por lo que se deci- 
dió mantener la búsqueda solo con una palabra para esta categoría, "incertidumbre". Hasta aquí se cumple que se cuente con artículos de la categoría $\mathbf{E}$ y $\mathbf{U}$. Para cumplir con la categoria $\mathbf{P}$, la revisión de tales palabras se realizó de manera visual. Esto debido a que tanto el programa The Shell como Xalok, permiten visualizar el artículo en la parte derecha de la pantalla. Una captura de pantalla del proceso de búsqueda mencionado se encuentra en el Anexo 1.

El mayor beneficio para la robustez del índice fue que, de manera inesperada, el analisis visual se convirtió en un proceso de auditoría de los artículos y así se desecharon artículos que no tenían relación con la búsqueda de fuentes de incertidumbre política, como son el horóscopo o artículos de fútbol los cuales no pueden filtrarse $a$ priori porque forman parte del archivo y sin embargo contenían algunas de las palabras clave.

Ya con los artículos con palabras clave EPU contados en un año específico, se procedió a contarlos por mes y a repetir el proceso para los 18 años restantes. De esta manera se obtuvo una primera serie llamada "conteo crudo". En el trabajo realizado por Perico (2018) se aumenta una categoría para capturar solo shocks de incertidumbre local. El hecho de aumentar esta palabra puede ser discutido.
Por un lado se intenta cuantificar la captura de eventos internacionales en la formación de opinión pública que pueda tener connotaciones inciertas, esto a través de la publicación de tales artículos en un periódico con alcance nacional. En segundo lugar y más importante, no todas las noticias en las que la fuente de incertidumbre sea local van a tener implícita la palabra "Ecuador", más aún si el periódico y los lectores son nacionales; esta palabra podría ser válida en publicaciones internacionales que necesiten especificar el lugar de los sucesos ocurridos.

Lo antes expuesto quizás pueda ser la razón por la que Baker, et al. (2016) y el índice desarrollado por Cerda, et al. (2018) para Chile, omitan una categoría con el nombre del país y se centren en palabras con las tres categorías EPU. Como evidencia sin embargo se presentan las dos series.

Una última consideración a tomar en cuenta es que, el proceso de búsqueda y conteo de artículos se lo realizó en las instalaciones del periódico a pesar de existir un archivo digital en la página web www.elcomercio.com desde el año 2003 ya que no todos lo artículos escritos se suben al internet, menos aún mientras más antigua se haga la búsqueda.

\section{Estadística descriptiva y transformaciones}


La primera transformación fue escalar la serie en función de todo el volumen de artículos escritos. Debido a que el número de artículos escritos en un día varía en función de la publicidad y la cantidad de eventos que sucedan, fue necesario realizar un conteo del total de artículos escritos mensualmente para así al dividir el "conteo crudo" para el núme- ro total de artículos escritos en un mes, obtener observaciones escaladas.

A las series escaladas se les dio el nombre de EPUs y EPUEs. La descripción de estas dos series juntos a las tres anteriores que generaron estos datos se presentan en las ecuaciones y tabla a continuación.

\section{Tabla 2. Descripción de variables}

\begin{tabular}{llllll}
\hline Variables & Obs & Media & Desv Est (s.d) & Min & Max \\
\hline EPUc & 228 & 16,197 & 8,023 & 0 & 44 \\
EPUEc & 228 & 7,973 & 5,141 & 0 & 26 \\
totalmonth & 228 & 1391,899 & 129,667 & 1065 & 2235 \\
EPUs & 228 & 0,011 & 0,006 & 0 & 0,032 \\
EPUEs & 228 & 0,006 & 0,004 & 0 & 0,178 \\
\hline
\end{tabular}

Elaborado por: Sebastián Padilla

Como se puede observar en la tabla descriptiva, en promedio se escribieron 16 noticias y un máximo de 44 al mes que reflejan incertidumbre. A su vez, estas noticias representaron en promedio el 1,1\% y un máximo del 3,2\% de los artículos publicados en sus respectivos meses.
En el siguiente gráfico se muestran las dos series escaladas. Por los argumentos expuestos con anterioridad respecto a la validez de incluir la categoría Ecuador, de aquí en adelante se utiliza la serie EPU para las transformaciones y posterior índice resultante. 


\section{Gráfico 1. Series escaladas EPUs y EPUEs}

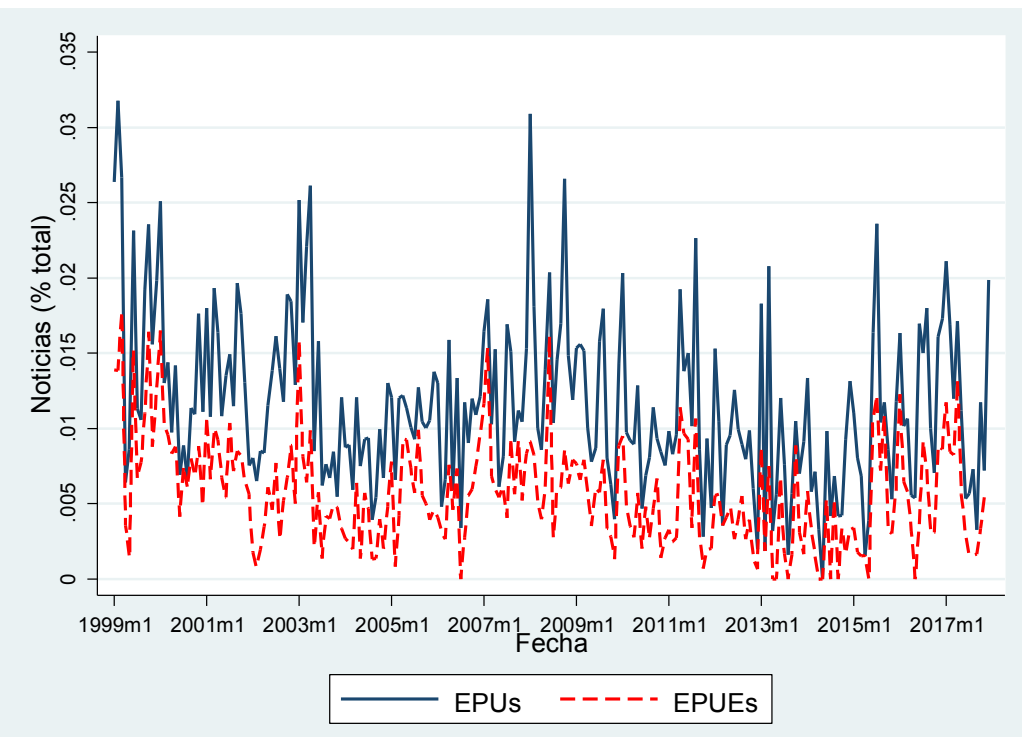

Elaborado por: Sebastián Padilla

Las transformaciones tratadas a continuación tienen como objetivo obtener una serie normalizada de media 100. Sin embargo, a diferencia de la investigación de Baker, et al. (2016) en el que utilizan 10 periódicos, en casos en los que la fuente de información es un solo periódico, como el de la presente investigación, el de Perico (2018) y el de Cerda, et al. (2018), estas transformaciones parecerían no ser necesarias.

En palabras de Stockburger (2016) la normalización de datos es necesaria al comparar un mismo fenó- meno en diferentes poblaciones con diferente tamaño, de lo contrario no se sabe la influencia de tal fenómeno en relación al número total de casos. A esto habría que añadir, situaciones en las que existan observaciones en diferentes escalas o unidades.

A pesar de aquello, se decidió seguir la metodología propuesta ya que permite una futura comparación tanto con índices de otros países como medidas con media 100, los proponentes de la metodología refieren: "We use the same approach for other countries and 
indexes" (Baker, et al., 2016). También se sigue la metodología a pesar de lo expuesto, porque el método de normalización al ser una transformación lineal, no altera la distribución de los datos (Stockburger, 2016).

En primer lugar se procedió con la estandarización de la serie escalada, diviendo EPU para su desviaciones estándar. De esta manera se obtuvo una nueva serie con desviación estándar unitaria, a la cual se la llamó EPUsd. En segundo lugar, se normalizó la serie di- viendo cada observación de EPUsd para su media y multiplicándola por 100. A esta serie normalizada con media 100 se la llamó EPUn.

$$
\mathrm{EPUsd}=\mathrm{EPUS} /\left(\sigma_{-} E P U s\right)
$$

$$
E P U n=(E P U s d \times 100) / \mu \_E P U s d
$$

En la siguiente tabla se observa la descripición de las variables transformadas junto con las originales.

\section{Tabla 3. Descripción de las variables transformadas}

\begin{tabular}{llllll}
\hline Variables & Obs & Media & Desv Est (s.d) & Min & Max \\
\hline EPUs & 228 & 0,011 & 0,006 & 0 & 0,032 \\
EPUsd & 228 & 2,056 & 1 & 0 & 5,635 \\
EPUn & 228 & 100 & 48,626 & 0 & 274,020
\end{tabular}

Elaborado por: Sebastián Padilla

\section{Resultados y sucesos históricos}

Antes de presentar los índices es pertinente identificar periodos clave a priori. Al menos tres eventos desde el año 1999 hasta el 2017 generaron incertidumbre en la economía ecuatoriana. El primero de ellos fue el feriado bancario y la consecuente dolarización del sistema monetario, eventos que se anunciaron en marzo de 1999 y enero del 2000 respectivamente, pero los cuales se habían forjado años atrás y sus consecuencias se sintieron también años después.
Un segundo evento de incertidumbre internacional fue la crisis de 2008 que tuvo origen internacional y se esparció por todo el mundo afectando a la economía ecuatoriana en 2009 así como a las demás economías latinoamericanas, aunque en menor medida. Como ejemplo, el 2009 la región de América Latina y el Caribe registró un crecimiento promedio económico de $-1,9 \%$ frente a 3,4 \% en 2008. Por su parte Ecuador creció en 2009 0,6 \% frente a 6,4\% en 2008. 
El tercer evento de incertidumbre fue la caída del precio de petróleo que inició en 2015 y que, entre varias consecuencias en la economía ecuatoriana, llevó a una recesión en el año 2016 con un crecimiento económico de -1,6\%.

Estos tres periodos han sido elegidos de manera subjetiva y son de ayuda para ubicar momentos en la historia reciente que deberían estar representa- dos de alguna manera en el índice. Cabe aclarar que hacer un juicio de valor acerca de las fuentes de incertidumbre de los periodos identificados o analizarlos a profundidad queda por fuera del alcance de la investigación.

A continuación, se presentan los resultados del índice EPU normalizado. En el Anexo 2 se encuentra una tabla con los valores del índice construido.

\section{Gráfico 3. Índice de incertidumbre de política económica Ecuador}

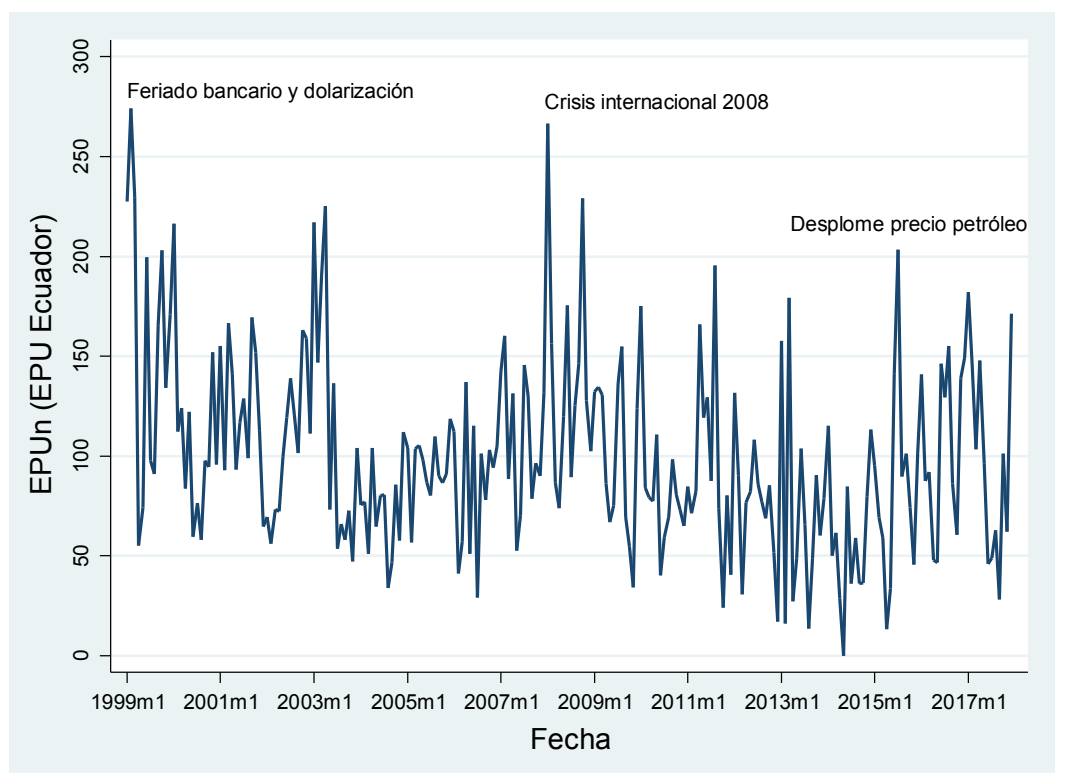

Elaborado por: Sebastián Padilla

Taly como se puede evidenciar en con anterioridad están representados el gráfico los tres eventos identificados en el índice por sobre los 200 puntos ex- 
cepto uno. Resalta de manera especial el incremento de incertidumbre de manera sostenida durante el año 2002, al pasar de 69,43 hasta un pico de 217,1 en enero de 2003. De acuerdo con las publicaciones de la fecha, varios eventos de distinta índole fueron noticia y pudieron haber influenciado el incremento del índice.

Entre ellos se registraron principalmente la construcción del Oleoducto de Crudos Pesados con una fuerte oposición de grupos ambientalistas y también de organizaciones campesinas, la erupción del volcán Reventador y el fracaso del Consejo Nacional de Modernización-CONAM en su afán de privatizar el sector eléctrico y de telecomunicaciones luego de haber sido declarada inconstitucional la ley Trole II.

En el 2011, la principal fuente de incertidumbre parece haber sido externa. Por un lado, la crisis del euro que comenzó en el año 2010 con la aprobación de un paquete de rescate de 750 mil millones de euros, crisis que alcanzó su cúspide a finales del 2011. Durante estos meses, según datos del Banco Central Europeo, los rendimientos de los bonos a largo plazo (cuya correlación es positiva con la percepción del riesgo) o también llamada tasa de interés a largo plazo de algunos países como Grecia, Italia, España y Portugal fueron las más altas desde que existe el euro.
Estos eventos sumados a la posible primera mora de pagos del gobierno estadounidense en la historia tuvieron impacto en la cobertura noticiosa sobre posibles implicaciones para el país en términos de remesas, inflación y exportaciones.

En el año 2013 el pico en los primeros meses puede estar relacionado con las elecciones en las que Alianza País obtuvo por primera vez desde el retorno a la democracia mayoría parlamentaria y Rafael Correa fue reelecto por segunda ocasión con el 60 \% de votos. Con esta mayoría el gobierno pudo finalmente aprobar en junio de 2013 la ley de comunicación a la que los medios como El Comercio se oponían.

Finalmente, el índice muestra el tercer evento de incertidumbre identificado a priori en el 2015, el cual parece extenderse hasta inicios del 2017. Según datos del Banco Central del Ecuador, durante este período el precio de barril llegó a cotizarse a \$25 por barril convirtiendo a la emisión de deuda en la principal fuente de ingreso del país, la deuda pública pasó de 27 \% PIB a inicios del 2015 a 46 \% PIB al cierre del 2017.

También en el 2016 se registró un terremoto de 7,8 grados en la escala de Richter. A nivel internacional los sucesos más importantes fueron la apreciación del dólar, la elección de Donald Trump 
como presidente de Estados Unidos, el acuerdo de paz entre Colombia y las FARC y un poco más lejano a la realidad ecuatoriana, el Brexit.

Es necesario aclarar que no hay manera de demostrar que efectivamente estos pocos eventos listados sean la causa del comportamiento de la serie, sin embargo, es un ejercicio de contextualización para establecer alguna posible relación entre ellos. Al menos en lo que respecta a un ejercicio de revisión histórica de eventos, el índice parece ser una variable proxy válida de la incertidumbre.

\section{Incertidumbre y otras medidas}

En un primer momento se evalúa el EPU con índices de incertidumbre para Estados Unidos y Colombia bajo la misma metodología, más adelante se hará lo mismo con el VIX (Chicago Board Options Exchange Market Volatility Index), el cual es una medida de volatilidad histórica calculada a partir del índice S\&P 500 y desde el lado de la teoría keynesiana, la tasa de variación interanual de depósitos a la vista. Una última comparación será llevada a cabo con un rezago del precio de petróleo WTI, esto debido a que se trata del principal producto de exportación con alrededor del $30 \%$ de la oferta exportable total de los últimos 20 años según información del BCE.

El principal socio comercial de Ecuador es Estados Unidos, alrededor del $38 \%$ de las exportaciones en los últimos 20 años de acuerdo con cifras del Banco Central del Ecuador tienen como destino EE. UU. A su vez, al ser el dólar la moneda de circulación en el Ecuador, las fluctuaciones en su tipo de cambio tienen efectos en el sector externo de la economía ecuatoriana.

A continuación se muestra el índice junto al de Estados Unidos realizado por Baker, et al. (2016). 


\section{Gráfico 4. EPU Ecuador y EE. UU}

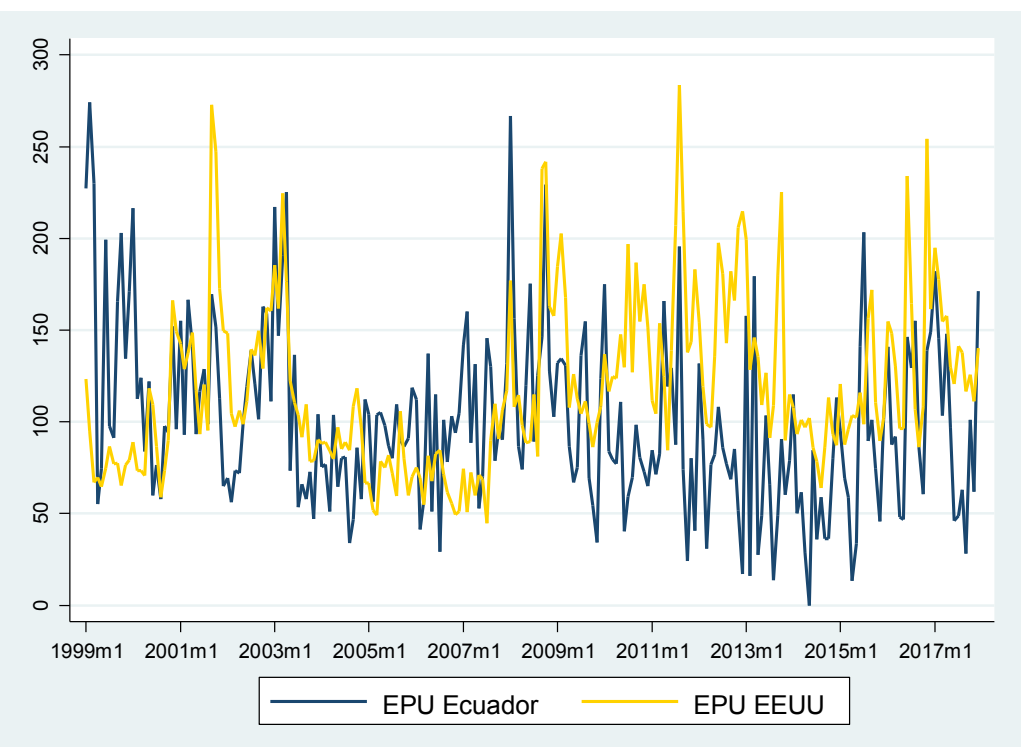

Elaborado por: Sebastián Padilla

Se observa que hasta el 2008 el EPU de EE. UU se mantiene en promedio por debajo del EPU para el Ecuador con un pico en 2001 cuando fue el ataque terrorista a las torres gemelas. A partir del 2009, la incertidumbre parece haber aumentado hasta llegar a los 283 puntos en mediados del 2011 con el riesgo de que la deuda de los Estados Unidos pudo haber caído en mora de pagos, evento identificado como "debt ceiling crisis" por parte de Baker, et al. (2016).
Colombia es el primer destino de las exportaciones industriales del Ecuador de acuerdo con cifras del Ministerio de Comercio Exterior. A esto se suma que los dos países vecinos enfrentan dificultades comunes en materia de seguridad en la frontera norte y están igual de expuestos frente a shocks internacionales.

A continuación se muestra el índice junto al de Colombia realizado por Perico (2018). 


\section{Gráfico 5. EPU Ecuador y Colombia}

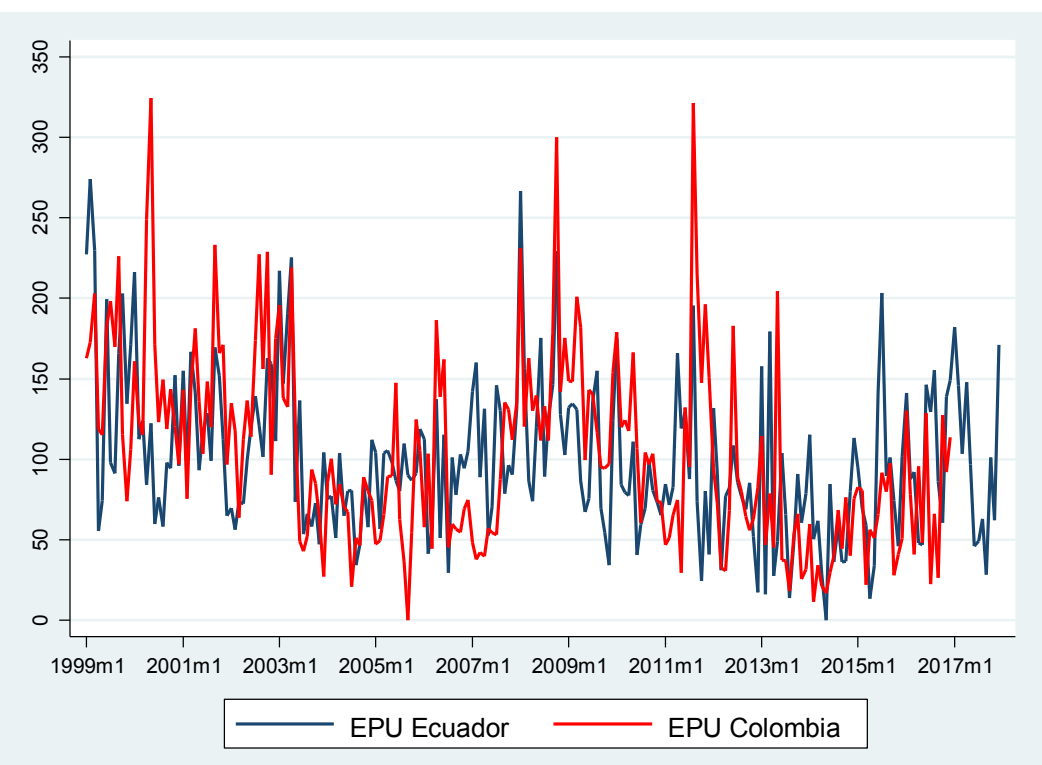

Elaborado por: Sebastián Padilla

A diferencia del Ecuador, los shocks de incertidumbre en Colombia parecen ser mucho más fuertes; mientras que la crisis economónica de 1999 es el evento que representa el mayor pico en Ecuador, Colombia registra tres eventos identificados por Perico (2018) que superan los 300 puntos. En el 2000 se registró la crisis del proceso de paz, la crisis del 2008 que se convirtió en una recesión lo cual no sucedió en Ecuador y en 2011 los mismos eventos internacionales que afectaron al Ecuador pero tu- vieron un mayor impacto en la redacción de artículos en Colombia.

Según lo expuesto en el trabajo de Baker, et al. (2016) el VIX tiene una correlación de 0,58 con su índice de incertidumbre para Estados Unidos. Se esperaría que en el caso ecuatoriano la correlación sea menor debido a que muchas de las empresas listadas en el S\&P 500 no tienen relación directa con la economía ecuatoriana.

El índice S\&P 500, formalmente llamado Standard y Poor's 500 Index es 
un índice ponderado de la capitalización bursátil de las 500 mayores empresas de Estados Unidos, independientemente del sector industrial en la que sen encuentran.

En todo caso, el VIX es la medida más antigua de incertidumbre que es- tima los efectos de ella en la economía real. A continuación se muestra el índice junto al VIX disponible en la página web del Banco de la Reserva Federal de St. Louis ${ }^{4}$.

\section{Gráfico 6. EPU Ecuador y VIX}

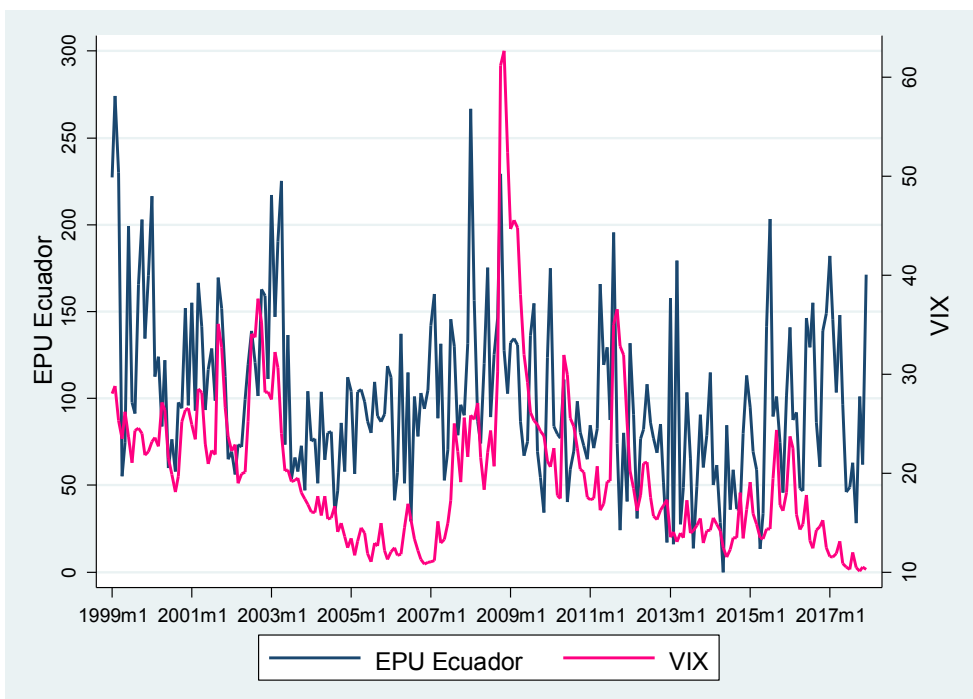

Elaborado por: Sebastián Padilla

El hallazgo más interesante es que hasta antes de la crisis del 2008, a inicios del 2007 el VIX se encontraba en sus niveles más bajos al mismo nivel que los últimos meses de 2017, entre 10 y 10,5. No parece haber relación en los picos más allá del período 2008-2009.

La siguiente comparación está hecha con la variación interanual de depósitos a la vista del sistema financiero. La teoría keynesiana relaciona períodos de incertidumbre con un incremento en la

\footnotetext{
${ }^{4}$ La serie VIX corresponde al promedio mensual de la serie original con periodicidad diaria.
} 
preferencia por liquidez por parte de los agentes económicos (Keynes, 1936). Esta preferencia por la liquidez podría traducirse en una disminución de los depósitos.

Debido a que la masa monetaria en términos corrientes aumenta con el tiempo y existen eventos estacionales que afectan los depósitos bancarios como pago de utilidades o navidad, la serie utilizada que evita ambos inconvenientes y permite comprender de mejor manera su comportamiento es la variación interanual de los depósitos a la vista publicada a nivel mensual por la Superintendencia de Bancos en su balance de bancos privados con datos desde el $2003^{5}$ y recopilada a su vez en el Boletín Monetario Semanal del BCE.

\section{Gráfico 7. EPU Ecuador y variación interanual de depósitos a la vista}

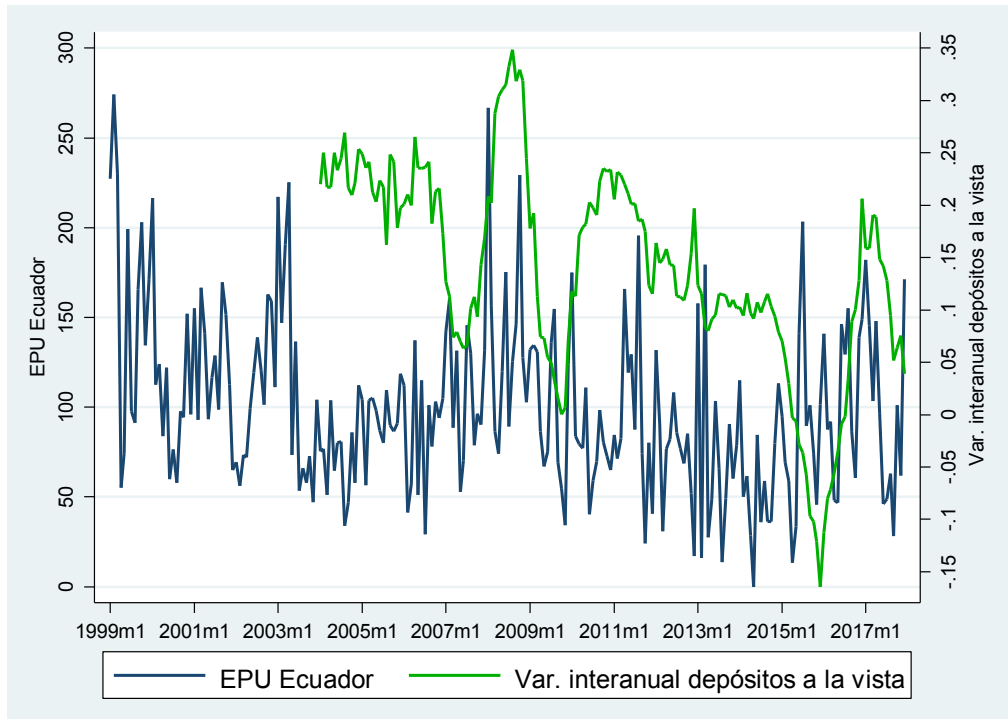

Elaborado por: Sebastián Padilla.

\footnotetext{
${ }^{5}$ La Superintendencia de Bancos en sus boletines de series de bancos privados incluye al Banco del Pacífico dentro del grupo. Dentro del boletín, en el Balance se encuentra la cuenta "Obligaciones con el público", e incluye por orden de liquidez: 1) Depósitos a la vista, 2) Operaciones de reporto, 3) Depósitos a plazo, 4) Depósitos de garantía y 5) Depósitos restringidos. En el Boletín Monetario Semanal del BCE esta información se encuentra recopilada dentro del Panorama Financiero.
} 
En el período analizado, la tasa promedio de crecimiento interanual de depósitos a la vista fue de 13,97\%. Existen dos momentos en los que la tasa de variación es negativa, a finales del 2009 durante dos meses muy cercana a cero y durante un año desde junio del 2015 a junio del 2016 con variaciones negativas de hasta $13 \%$.

El primer evento podría estar relacionado con la crisis del 2008, sin embargo, durante gran parte de ese año las tasas de crecimiento fueron las más altas registradas, llegando a 33,8\%. En el período del 2015 al 2016 parece que los depósitos tuvieron una reacción negativa más fuerte que el EPU frente a la coyuntura de disminución del precio del petróleo.

La comparación del índice de incertidumbre frente a un rezago del precio del petróleo WTI, está motivada porque se trata del principal producto de exportación del Ecuador y asumiendo que la producción no varía en el corto plazo, el precio podría ser un indicador adelantado del índice de incertidumbre.

\section{Gráfico 8. EPU Ecuador y un rezago del precio del petróleo}

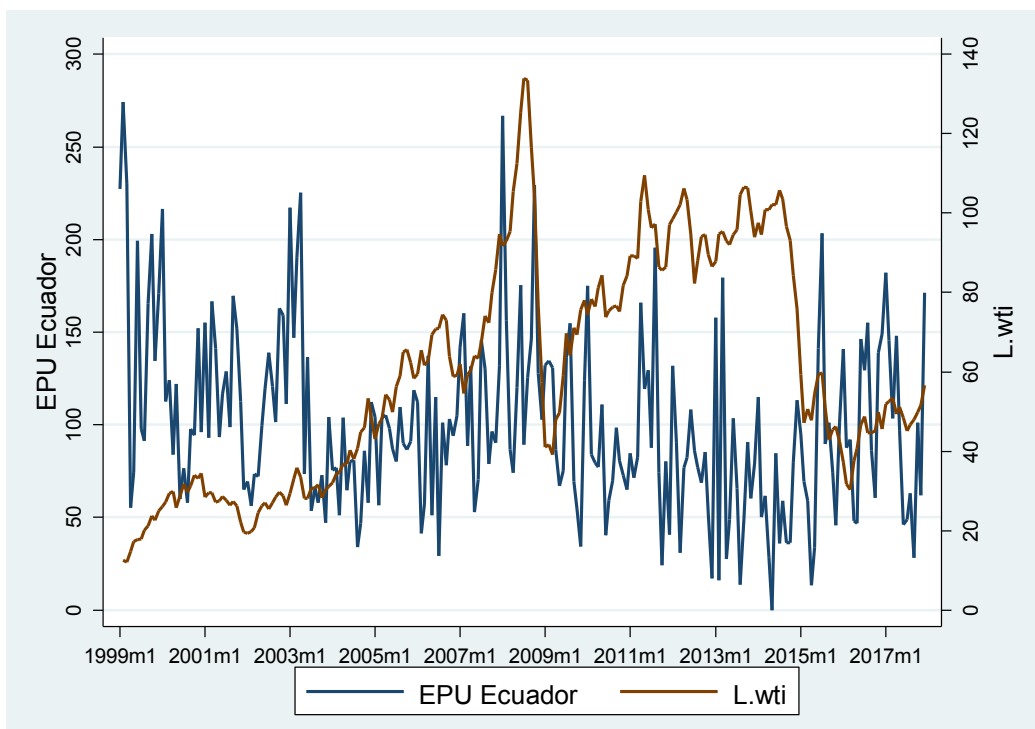

Elaborado por: Sebastián Padilla 
De manera visual, se puede evidenciar tres comportamientos en el gráfico. Hasta el año 2003 y desde el 2009 al 2015 una relación aparentemente negativa entre las series, es decir una disminución del precio del petróleo asociado con un incremento en el índice de incertidumbre y viceversa; del 2003 al 2009 una relación positiva y desde el 2015 una falta de relación aparente.

Se esperaría que tanto los índices de incertidumbre de Estados Unidos, Colombia y el VIX estén correlacionados de manera positiva con el EPU Ecuador y de manera negativa con la variación de depósitos. Para corroborar la existencia de relaciones entre las series analizadas de manera visual, a continuación, se presenta la matriz de correlación de Pearson.

Se utiliza la correlación de Pearson con el método pairwise deletion de tratamiento de datos perdidos ya que la serie de variación de depósitos a la vista tiene datos desde el 2003 y el EPU Colombia tiene datos hasta el 2016; la diferencia entre las dos maneras de tratar con datos perdidos, pairwise y casewise da como resultado coeficientes ligeramente diferentes de acuerdo con Howell (2008).

En un conjunto de datos con tres variables $x_{1}, x_{2}, x_{3}$ en la que $x_{2}$ tiene la mitad del número observaciones de las otras dos, el coeficiente de correlación de Pearson bajo casewise entre $x_{1}$ y $x_{3}$ se realiza con las observaciones válidas para todo el conjunto de variables, es decir la mitad de las observaciones debido a $x_{2}$. En cambio, el coeficiente con pairwise realiza el cálculo para las variables de manera separada, e incluye todas las observaciones válidas para cada comparación, así el número de observaciones de $x_{2}$ no influye en el cálculo de la correlación entre $x_{1}$ y $x_{3}$ (Howell, 2008). 
Tabla 4. Matriz de correlaciones de Pearson

\begin{tabular}{|c|c|c|c|c|c|c|}
\hline Corr, $p$-value, obs. & EPU EC & EPU US & EPU COL & VIX & DEPOS & L.WTI \\
\hline \multirow[t]{2}{*}{ EPU EC } & 1 & & & & & \\
\hline & 228 & & & & & \\
\hline \multirow[t]{3}{*}{ EPU US } & $0,2435^{*}$ & 1 & & & & \\
\hline & 0.0002 & & & & & \\
\hline & 228 & 228 & & & & \\
\hline \multirow[t]{3}{*}{ EPU COL } & $0,4960^{*}$ & $0,3689^{*}$ & 1 & & & \\
\hline & 0.0000 & 0.0000 & & & & \\
\hline & 216 & 216 & 216 & & & \\
\hline \multirow[t]{3}{*}{ VIX } & $0,3359^{*}$ & $0,4330^{*}$ & $0,6597 *$ & 1 & & \\
\hline & 0.0000 & 0.0000 & 0.0000 & & & \\
\hline & 228 & 228 & 216 & 228 & & \\
\hline \multirow[t]{3}{*}{ DEPOS } & 0.1103 & $-0,0149$ & 0.2375 & 0.1635 & 1 & \\
\hline & 0.1545 & 0.8475 & 0.0028 & 0.0342 & & \\
\hline & 168 & 168 & 156 & 168 & 168 & \\
\hline \multirow[t]{3}{*}{ L.WTI } & $-0.1925 *$ & $0.1394^{*}$ & $-0.224 *$ & $-0.1369 *$ & $0.3006 *$ & 1 \\
\hline & 0.0036 & 0.0358 & 0.0010 & 0 & 0.0001 & \\
\hline & 227 & 227 & 215 & 227 & 168 & 227 \\
\hline
\end{tabular}

* Significativo al $95 \%$ nivel de confianza

Elaborado por: Sebastián Padilla

Un coeficiente de correlación entre dos variables debe reflejar la dirección, medida por el signo, y la magnitud de esta con un determinado nivel de significancia. En la matriz, todas las correlaciones significativas tienen la dirección esperada. El coeficiente de correlación más alto $(0,50)$ es entre EPU de Ecuadory EPU Colombia.

La correlación entre la tasa de variación interanual de depósitos a la vista y el índice de incertidumbre, que se es- peraría fuera negativa, no tiene la dirección esperada pero no es significativa ni al $90 \%$ de nivel de confianza.

Existe una correlación significativa pero baja entre EPU y un rezago del precio del petróleo $(-0,19)$, la dirección es la que se esperaría si el precio del petróleo es de alguna forma un indicador adelantado de la percepción de incertidumbre calculada. 


\section{CONCLUSIONES}

Frente a la creciente disponibilidad de datos, es posible aportar con evidencia para definiciones que en su tiempo no pudieron ser cuantificables como es la incertidumbre a la que se refería Knight o Keynes. Específicamente se deja de lado las probabilidades tanto objetivas como subjetivas, que son la base de la inconmensurabilidad de la incertidumbre y se presenta una medida innovadora para la teoría keynesiana.

A su vez, el índice constituye una fuente de evidencia acerca de la naturaleza impredecible de los sistemas económicos ya que en todo momento la incertidumbre capturada es positiva y oscila alrededor de su media. No existen, por lo tanto, ambientes de incertidumbre sino un estado incierto permanente en el que los individuos y las firmas deben tomar decisiones tal y como la teoría keynesiana argumenta.

La gran diferencia transversal de los aportes presentados parecería ser la naturaleza normativa o descriptiva de las ideas presentadas. La hipótesis de expectativas racionales es una hipótesis de trabajo para modelos específicos. En muchos casos, los resultados de estos modelos pasan a ser parte de la teoría económica y por lo tanto dejan de ser específicos. Sin embargo, parecería ser que las predicciones de las variables y las acciones de política económica no pueden realizarse en base a un comportamiento ideal de los individuos sino al real.

En cuanto a la utilidad esperada, los aportes de Savage en ampliar la comprensión de actos, estados y resultados por un lado brindan mayor libertad a que los individuos formen probabilidades subjetivas, esto a su vez reduce su teoría a lo que él llamó problemas simples de decisión.

La variable proxy construida parece responder bien a una revisión no tan profunda de eventos históricos caracterizados por altos niveles de incertidumbre, globales y locales, que fueron identificados antes y después de construir la serie.

En la comparación con otras medidas de incertidumbre se encontraron correlaciones positivas significativas que van desde 0,24 hasta 0,50, una correlación negativa significativa con un rezago del precio del petróleo $(-0,19)$ y la inexistencia de una correlación con la variación de depósitos a la vista que se esperaría fuera negativa para el período analizado. 


\section{REFERENCIAS BIBLIOGRÁFICAS}

Arellano, C., Yan, B., \& Kehoe, P. (2016). Financial Frictions and Fluctuations in Volatility. Federal Reserve Bank of Minneapolis.

Bachmann, R., Elstner, S., \& Eric, S. (2013). Uncertainty and Economic Activity: Evidence from Business Survey Data. American Economic Journal: Macroeconomics, 5(2), 217-249.

Baker, S., Bloom, N., \& Davis, S. (2016). Mesauring Economic Policy Uncertainty. The Quarterly Journal of Economics, 1593-1636.

Bernanke, B. (1983). Irreversibility, Uncertainty, and Cyclical Investment. The Quarterly Journal of Economics, 97(1), 85-106.

Blanchard, O., Amighini, A., \& Giavazzi, F. (2012). Macroeconomía. Madrid: Pearson Educación, S.A.

Bloom, N. (2009). The Impact of Uncertainty Shocks. Econometrica, 77(3), 623-685.

Briggs, R. (2017). Normative Theories of Rational Choice: Expected Utility. The Stanford Encyclopedia of Philosophy.

Carrière-Swallow, Y., \& Céspedes, L. F. (2013). The impact of uncertainty shocks in emerging economies. Journal of International Economics.

Cerda, R., Silva, Á., \& Valente, J. (2018). Im- pact of economic uncertainty in a small open economy: the case of Chile. Journal of Applied Economics, 2894-2908.

Chicago Board Options Exchange. (2018). Federal Reserve Bank of St. Louis. Obtenido de Economic Research Division: https://fred.stlouisfed. org/series/VIXCLS

D'Amico, S., \& Orphanides, A. (2008). Uncertainty and disagreement in economic forecasting. Board of Governors of the Federal Reserve System.

Danziger, S., Van der Gagg, J., Smolensky, E., \& Tausigg, M. (1982). The life cycle hypothesis and the consumption behavior of the elderly. Journal of Post Keynesian Economics.

Davidson, P. (1991). Is Probability Theory Relevant for Uncertainty? Journal of Economic Perspectives, 129-143. Davidson, P. (1994). Postkeynesian Macroeconomic Theory (Vol. 2). Cambridge: Edward Elgar.

Davidson, P. (2002). Financial markets, money and the real world. Northampton: Edward Elgar Publishing, Inc.

Ferrari-Filho, F., \& Conceicao, O. (2005). The Concept of Uncertainty in Post Keynesian Theory and in Institutional Economics. Journal of 
Economic Issues, 34(3).

Howell, D. C. (2008). The Treatment of Missing Data. En W. Outhwaite, \& S. Turner, Handbook of Social Science Methodology. London: Sage. Jurado, K., Ludvigson, S., \& Serena, N. (2015). Measuring Uncertainty. American Economic Review, 105(3), 1177-1216.

Keynes, J. M. (1936). The General Theory of Employment, Interest and Money. Londres: Macmillan.

Keynes, J. M. (1937). The General Theory of Employment. The Quarterly Journal of Economics, 209-223.

Knight, F. (1921). Risk, Uncertainty, and Profit. Boston: Houghton Mifflin Co.

Lucas, R., \& Sargent, T. (1981). Rational Expectations and Econometric Practice. Minneapolis: The University of Minnesota Press.

Machina, M. (1987). Choice Under Uncertainty: Problems Solved and Unsolved. Journal of Economic Perspectives, 121-154.

Muth, J. (1961). Rational Expectations and the Theory of Price Movements. Econometrica, 29(6).

Perico Ortiz, D. (2018). Measuring Eco- nomic Policy Uncertainty in Colombia: a News Based Approach. http://www.policyuncertainty. com/colombia_monthly.html.

Sargent, T., \& Wallace, N. (1975). "Rational" Expectations, the Optimal Monetary Instrument, and the Optimal Money Supply Rule. Journal of Political Economy, 88(2).

Sargent, T., \& Wallace, N. (1976). Rational Expectations and the Theory of Economic Policy. Journal of Monetary Economics, 2.

Savage, L. (1954). The Foundations of Statistics. New York: Wiley.

Stockburger, D. (2016). Introductory to Statistics: Concepts, Models and Applications. St. Louis: Missouri State University.

Superintendencia de Bancos. (2018). Portal Estadístico. Obtenido de Series Bancos Privados: http://estadisticas.superbancos.gob.ec/portalestadistico/portalestudios/?page_id=791

Wooldridge, J. (2012). Introductory Econometrics: A Modern Approach. Mason, Ohio: South-Western Cengage Learning. 


\section{ANEXOS}

\section{ANEXO 1. BÚSQUEDA DE ARTÍCULO EPU}

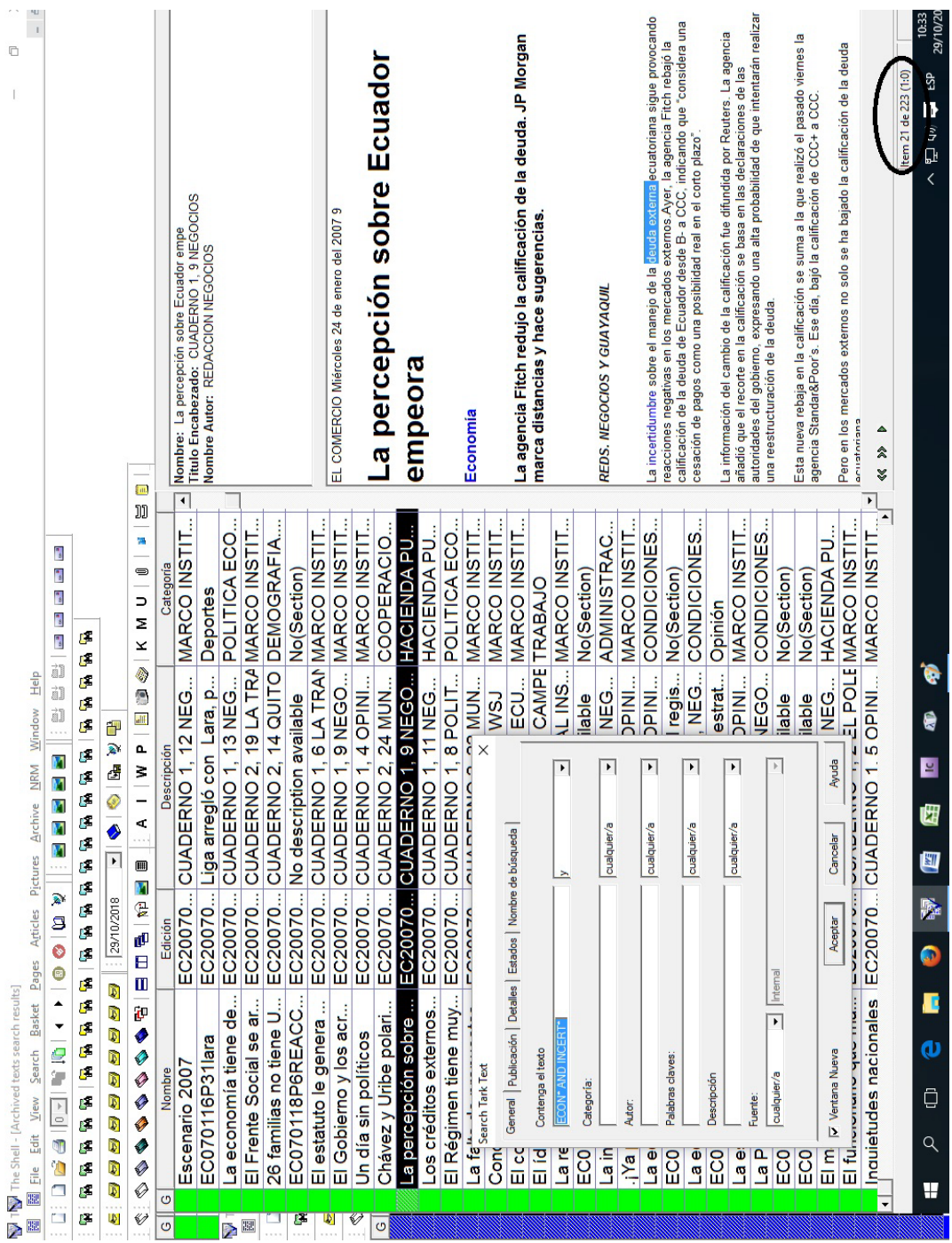




\section{ANEXO 2. EPU ECUADOR MENSUAL}

ํํำ ㅇำ

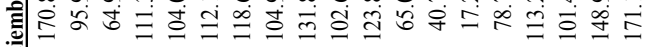

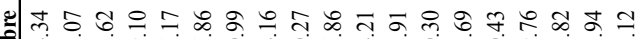

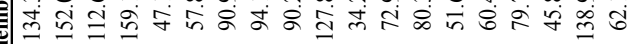

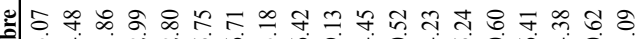

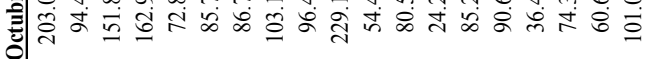

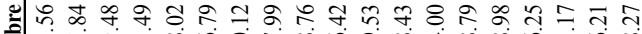
㝵

ํำ

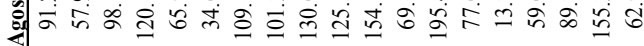

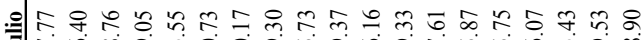
छ

ㅇำ

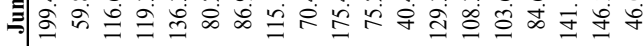

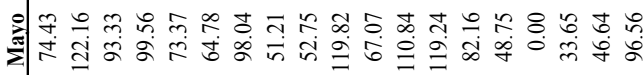

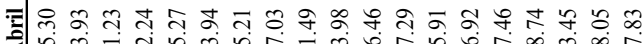

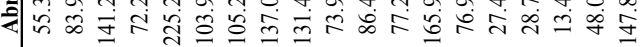

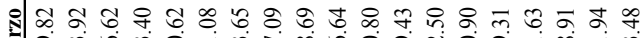

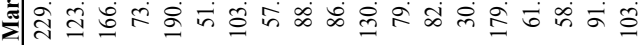

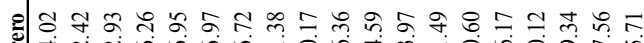

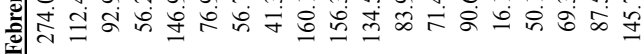

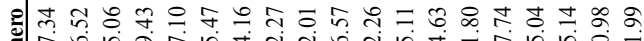

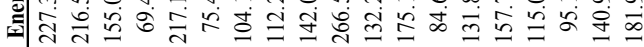

